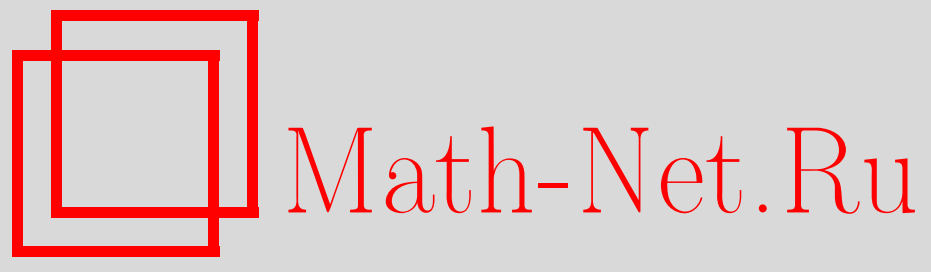

Д. И. Савельев, Сообщение об игре на универсуме множеств, Матем. заметки, 2007, том 81, выпуск 5, 797-800 DOI: https://doi.org/10.4213/mzm3727

Использование Общероссийского математического портала Math-Net.Ru подразумевает, что вы прочитали и согласны с пользовательским соглашением http://www . mathnet.ru/rus/agreement

Параметры загрузки:

IP: 54.162 .27 .143

26 апреля 2023 г., 03:44:12 


\section{Сообщение об игре на универсуме множеств}

\section{Д. И. Савельев}

Мы работаем в теории множеств Цермело-Френкеля ZF без аксиомы регулярности $\mathrm{AR}$; мы не используем аксиому выбора AC, исключая лишь один случай. Пусть $\mathrm{ZF}^{-}$ и $\mathrm{ZFC}^{-}$- это ZF и ZFC без $\mathrm{AR}$. Обозначения, общепринятые в теории множеств (см. [1]): $V$ - универсум, т.е. класс всех множеств, $O n-$ класс всех ординалов, $V_{\alpha}-\alpha$-й уровень кумулятивной иерархии, tc - транзитивное замыкание, $\mathrm{cf}$ - конфинальность, и т.д. Пусть также $V_{\infty}$ обозначает класс всех фундированных множеств и $r$ - функцию ранга.

Предположим, что два игрока, I и II, начиная с данного множества $x$, пытаются так построить $\in$-убывающую последовательность множеств $x_{n}$, чтобы противник не смог продолжить ее: вначале I выбирает $x_{0} \in x$, затем II выбирает $x_{1} \in x_{0}$, и т.д.; игрок выигpъьвает, если противник не может сделать никакого следующего хода, т.е. если он смог выбрать пустое множество. (Эту игру - но не наши результаты - можно найти в [2], где она рассматривается в теории Куайна NF (изложение содержит неточности); кроме того, близкая игра упоминается в [3].) Назовем множество выигрышным, если оно допускает выигрышную стратегию для некоторого игрока. Пусть $W$ - класс всех выигрышных

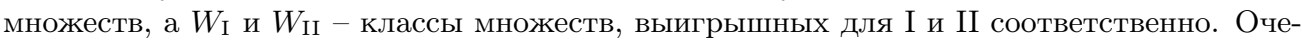
видно, $W_{\mathrm{I}} \cup W_{\mathrm{II}}=W$ и $W_{\mathrm{I}} \cap W_{\mathrm{II}}=0$. Класс $W$ имеет естественную иерархию: пусть $\gamma$ - ординал; назовем множество $2 \gamma$-выигрышным, если каждый его элемент является $(2 \delta+1)$-выигрышным для некоторого $\delta<\gamma$, и $(2 \gamma+1)$-выигрышным, если оно содержит $2 \gamma$-выигрышный элемент. Пусть $W_{\nu}-$ класс всех $\nu$-выигрышных множеств. Очевидно, $W_{0}=\{0\}, W_{2 \gamma+1}=V \backslash \mathscr{P}\left(V \backslash W_{2 \gamma}\right), W_{2 \gamma}=\mathscr{P}\left(\bigcup_{\delta<\gamma} W_{2 \delta+1}\right)$ и $W=\bigcup_{\nu \in O n} W_{\nu}$. Также $W_{\mathrm{I}}=V \backslash \mathscr{P}\left(V \backslash W_{\mathrm{II}}\right)=\bigcup_{\gamma \in O n} W_{2 \gamma+1}$ и $W_{\text {II }}=\mathscr{P}\left(W_{\mathrm{I}}\right)=\bigcup_{\gamma \in O n} W_{2 \gamma}$. Пусть $S_{\nu}-$ $\nu$-й уровень выигрышной иерархии: $S_{\nu}=W_{\nu} \backslash \bigcup_{\mu<\nu} W_{\mu}$. Очевидно, $W_{2 \gamma}=\bigcup_{\delta \leqslant \gamma} S_{2 \delta}$ и $W_{2 \gamma+1}=\bigcup_{\delta \leqslant \gamma} S_{2 \delta+1}$. Классы $S_{\nu}$ допускают рекуррентное определение:

$$
\begin{aligned}
S_{2 \gamma+1} & =\mathscr{P}\left(V \backslash \bigcup_{\delta<\gamma} S_{2 \delta}\right) \backslash \mathscr{P}\left(V \backslash S_{2 \gamma}\right), \\
S_{2 \gamma} & =\mathscr{P}\left(\bigcup_{\delta<\gamma} S_{2 \delta+1}\right) \backslash \bigcup_{\varepsilon<\gamma} \mathscr{P}\left(\bigcup_{\delta<\varepsilon} S_{2 \delta+1}\right) .
\end{aligned}
$$

Для $x \in W$ положим $w(x)=\min \left\{\nu: x \in S_{\nu}\right\}$. Если $x$ непусто,

$$
w(x)= \begin{cases}\min \left\{w\left(x_{0}\right)+1: x_{0} \in x \cap W_{\mathrm{II}}\right\}, & \text { если } x \in W_{\mathrm{I}}, \\ \sup \left\{w\left(x_{0}\right)+1: x_{0} \in x\right\}, & \text { если } x \in W_{\mathrm{II}}\end{cases}
$$

ЛЕмма 1. $S_{\nu}$ непусты для всех $\nu$.

Пусть $H W$ - класс всех наследственно выигрышных множеств: $H W=\{x: \operatorname{tc}(\{x\}) \subseteq$ $W\}$.

Лемма 2. Имеем $\mathscr{P}(W) \subseteq W$ u $\mathscr{P}(H W)=H W$.

Теорема 1. HW- внутренняя модель $\mathrm{ZF}^{-}$. Более того, $H W \supseteq V_{\infty}$.

Какие из классов $V \supseteq W \supseteq H W \supseteq V_{\infty}$ могут не совпадать? Утверждение 1) леммы 3 говорит, что достаточно знать ответ для $V \supseteq H W$ и $H W \supseteq V_{\infty}$, а теорема 4 далее - что все четыре остающихся случая непротиворечивы.

Работа выполнена при частичной поддержке Российского фонда фундаментальных исследований, грант № 06-01-00608-а. 
Лемма 3. 1) $V=W$ равносильно $W=H W$.

2) Если $V \backslash W$ непуст, то $V \backslash W u W \backslash H W$ собственные.

3) Если $H W \backslash V_{\infty}$ непуст, то он собственный.

4) AR равносильно $W=V_{\infty}$.

Как много $\nu$-выигрышных фундированных множеств с данным $\nu$ имеют данный ранг? Пусть $S_{\alpha, \nu}=V_{\alpha} \cap S_{\nu}$, или рекуррентно

$$
\begin{aligned}
S_{\alpha+1,2 \gamma+1} & =\mathscr{P}\left(V_{\alpha} \backslash \bigcup_{\delta<\gamma} S_{\alpha, 2 \delta}\right) \backslash \mathscr{P}\left(V_{\alpha} \backslash S_{\alpha, 2 \gamma}\right), \\
S_{\alpha+1,2 \gamma} & =\mathscr{P}\left(\bigcup_{\delta<\gamma} S_{\alpha, 2 \delta+1}\right) \backslash \bigcup_{\varepsilon<\gamma} \mathscr{P}\left(\bigcup_{\delta<\varepsilon} S_{\alpha, 2 \delta+1}\right) .
\end{aligned}
$$

Лемма 4. 1) Для всех фундированных $x$ выполнено $w(x) \leqslant r(x)$.

2) $S_{\alpha, \nu}$ непуст, если и только если $\nu<\alpha$.

3) $S_{\alpha+1, \nu} \backslash S_{\alpha, \nu}$ непуст, если и толъко если $0=\nu=\alpha$ или $0<\nu \leqslant \alpha$.

4) $S_{\alpha, \nu} \in S_{\alpha+1, \nu+1} \backslash S_{\alpha, \nu+1}$, если и только если $0=\nu<\alpha=1$ или $0<\nu<\alpha$.

Лемма 5. Пусть в $s_{\alpha, \nu}$ всегда $\alpha>\nu$ и, кроме случал 1), $\nu>0$. Тогда

1) (тривиальный случай) для всех $\alpha$ верно $\left|S_{\alpha, 0}\right|=1$;

2) (конечный случай) для всех $m<\omega$ верно

$$
\begin{aligned}
& \left|S_{m+1,2 k+1}\right|=2^{\left|V_{m}\right|-\sum_{j<k}\left|S_{m, 2 j}\right|}-2^{\left|V_{m}\right|-\sum_{j \leqslant k}\left|S_{m, 2 j}\right|}, \\
& \left|S_{m+1,2 k+2}\right|=2^{\sum_{j \leqslant k}\left|S_{m, 2 j+1}\right|}-2^{\sum_{j<k}\left|S_{m, 2 j+1}\right|} ;
\end{aligned}
$$

3) (бесконечный случай) для всех $\alpha \geqslant \omega$ верно $\left|S_{\alpha, \nu}\right|=\left|V_{\alpha}\right| u\left|S_{\alpha+1, \nu} \backslash S_{\alpha, \nu}\right|=\left|V_{\alpha+1}\right|$.

Это приводит к интересному вероятностному результату. Пусть

$$
\operatorname{Pr}\left(S_{\omega, n}\right)=\lim _{m \rightarrow \omega} \frac{\left|S_{\omega, n} \cap V_{m}\right|}{\left|V_{m}\right|}
$$

Легкие вычисления показывают, что эти пределы существуют (и Рr порождает вероятность на $\left.V_{\omega}\right)$; более того, имеет место

Теорема 2. Имеем $\operatorname{Pr}\left(S_{\omega, 1}\right)=\operatorname{Pr}\left(S_{\omega, 3}\right)=1 / 2\left(u \operatorname{Pr}\left(S_{\omega, n}\right)=0\right.$ для прочих $\left.n\right)$.

То есть "половина" наследственно конечных фундированных множеств состоит из 1-выигрышных множеств, а другая "половина" - из множеств 3-, но не 1-выигрышных. Как следствие, игрок I почти всегда имеет выигрышную стратегию, причем может выиграть очень быстро: за 1 или 3 хода.

Насколько класс $W$ похож на модель ZFC? Можно показать, что или $W=V$, или $W$ не может быть моделью многих аксиом ZFC. Неожиданно, что AR не входит в их число: $\mathrm{AR}^{W}$ совместима с $\neg \mathrm{AR}$. Пусть $\varsigma(C)$ означает "некоторое $x \in C$ не имеет $\in$-минимальных элементов".

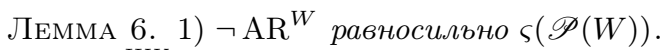

2) $\neg \mathrm{AR}^{H W}$ равносилвно $(H W)$.

3) $\mathrm{AR}^{H W}$ равносильно $H W=V_{\infty}$.

4) $\mathrm{AR}^{W}$ влечет $\mathrm{AR}^{H W}$.

Для результатов о непротиворечивости мы предлагаем новую конструкцию нефундированных моделей (см. также [4]). Обычное построение (основанное на факторизации по бисимуляциям; см.[3], [5]-[7]) приводит к моделям, слишком "нерасслоенным" для нас. Наша конструкция больше напоминает кумулятивную иерархию: мы берем подходящий $\left(M_{0}, E_{0}\right)$ в качестве начального уровня и применяем итерацию определенного 
аналога операции $\mathscr{P}$. В полученной иерархии верхние уровни будут концевыми расширениями нижних; интересующие нас свойства отражаются от нижних уровней и поэтому легко контролируемы. Скажем, что $v \in A$ представляет множество $y \subseteq A$ в $(A, R)$, если $y=\{u: u R v\}$, и что $(A, R)$ густой, если для любого множества $y \subseteq A$ существует $v \in A$, представляющий $y$ в $(A, R)$. Пусть $\mathscr{S}(A) \subseteq \mathscr{P}(A)$ состоит из всех непустых подмножеств класса $A$, кроме представленных в $(A, R)$. Пусть $B \supseteq A$. $(B, S)$ - концевое расширение $(A, R)$, если $S \cap(B \times A)=R$. $(A, R)$ отражсает формулу $\varphi$ над $(B, S)$, если $\varphi^{A, R}(x, \ldots) \leftrightarrow \varphi^{B, S}(x, \ldots)$ для всех $x, \ldots \in A$. Для данного $\left(M_{0}, E_{0}\right)$ определяем $\left(M_{\alpha}, E_{\alpha}\right)$ рекурсией по $\alpha$ :

$$
\begin{gathered}
M_{\alpha+1}=M_{\alpha} \cup \mathscr{S}\left(M_{\alpha}\right), \quad E_{\alpha+1}=E_{\alpha} \cup\left(\in \cap\left(M_{\alpha} \times \mathscr{S}\left(M_{\alpha}\right)\right)\right), \\
M_{\alpha}=\bigcup_{\beta<\alpha} M_{\beta}, \quad E_{\alpha}=\bigcup_{\beta<\alpha} E_{\beta}, \quad \text { если } \alpha \text { предельно, } \\
M=\bigcup_{\alpha \in O n} M_{\alpha}, \quad E=\bigcup_{\alpha \in O n} E_{\alpha} .
\end{gathered}
$$

В действительности, для нас важны $\left(M_{0}, E_{0}\right)$ со свойствами (i)-(iv):

(i) $\left\{u: u E_{0} v\right\}$;

(ii) $x \notin \operatorname{tc}(y)$, если $x, y \in M_{0}$;

(iii) $\left(M_{0}, E_{0}\right) \models$ "существует пустое множество";

(iv) $\left(M_{0}, E_{0}\right) \models$ аксиома экстенсиональности.

Лемма 7. Пусть $\left(M_{0}, E_{0}\right)$ удовлетворяет (i), (ii). Тогда

1) $(M, E)$ - концевое расширение каждого $\left(M_{\alpha}, E_{\alpha}\right)$;

2) $\left(M_{0}, E_{0}\right)$ отражает аксиому экстенсиональности над $(M, E)$;

3) если $\left(M_{0}, E_{0}\right)$ удовлетворяет также (iii), то $(M, E)$ густой;

4) $\left(M_{1}, E_{1}\right)$ отражает $\varsigma\left(W_{2 \gamma}\right)$ и $\varsigma\left(W_{\mathrm{II}}\right)$ над $(M, E)$;

5) $\left(M_{1}, E_{1}\right)$ отражает $\mathrm{AR}^{W}$ над $(M, E)$;

6) $(M, E) \mid=\varsigma\left(S_{2 \gamma}\right)$ влечет $\left(M_{1}, E_{1}\right) \models \varsigma\left(S_{2 \delta}\right)$ для некоторого $\delta \leqslant \gamma$.

Мы нуждаемся в 4)-6) только для результатов о выигрышности; согласно [8] для выполнения аксиом $\mathrm{ZF}^{-}$достаточно иметь 1)-3).

Теорема 3. Если $\left(M_{0}, E_{0}\right)$ удовлетворяет (i)-(iv), то $(M, E)$ - модель $\mathrm{ZF}^{-}$. Более того, это модель $\mathrm{ZFC}^{-}$(если $V$ - модель $\left.\mathrm{ZFC}^{-}\right)$.

Tеорема 4. Все непротиворечивые соотношения между $V, W, H W, V_{\infty}$ и релятивизациями AR на них - это в точности следующие пятъ:

1) $V=W=H W=V_{\infty}+\mathrm{AR}+\mathrm{AR}^{W}+\mathrm{AR}^{H W}$;

2) $V \neq W \neq H W=V_{\infty}+\neg \mathrm{AR}+\mathrm{AR}^{W}+\mathrm{AR}^{H W}$;

3) $V \neq W \neq H W=V_{\infty}+\neg \mathrm{AR}+\neg \mathrm{AR}^{W}+\mathrm{AR}^{H W}$;

4) $V=W=H W \neq V_{\infty}+\neg \mathrm{AR}+\neg \mathrm{AR}^{W}+\neg \mathrm{AR}^{H W}$;

5) $V \neq W \neq H W \neq V_{\infty}+\neg \mathrm{AR}+\neg \mathrm{AR}^{W}+\neg \mathrm{AR}^{H W}$.

Более тонкие результаты о $\varsigma(C)$ для различных $C \subseteq W$ следующие.

Лемма 8. 1) $\varsigma\left(W_{\mathrm{I}}\right)$ равносильно $\neg \mathrm{AR}$.

2) $\varsigma\left(W_{\text {II }}\right)$ влечет $\neg \mathrm{AR}^{W}$.

3) $\neg \varsigma\left(W_{\mathrm{II}}\right)$ совместимо $c \neg \mathrm{AR}^{W}$.

4) $\varsigma\left(W_{\text {II }}\right)$ непротиворечиво.

5) $\neg \varsigma\left(\mathscr{P}\left(W_{\text {II }}\right)\right)$. 
Лемма 9. 1) $\varsigma\left(S_{\nu}\right)$ влечет $\nu>1$.

2) $\neg \mathrm{AR}$ влечет $\varsigma\left(S_{\nu}\right)$ для всех нечетных $\nu>1$.

3) Если н четно, $\varsigma\left(S_{\mu}\right)$ влечет $\varsigma\left(S_{\nu}\right)$ для всех $\nu \geqslant \mu$.

4) Если $\mu$ предельно, $\varsigma\left(S_{\mu}\right)$ влечет $\varsigma\left(S_{\nu}\right)$ для некоторого $\nu \leqslant \mu, \operatorname{cf} \nu=\omega$.

Tеорема 5. Пусть $A \subseteq$ On. Тогда $\left\{\nu: \varsigma\left(S_{\nu}\right)\right\}=A$ совместимо с $\mathrm{ZFC}^{-}$в точности в одном из трех случаев:

1) A nycmo;

2) $A=\{\nu>1: \nu$ нечетно $\}$;

3) $A=\{\nu>1: \nu$ нечетно или $\nu \geqslant \mu\}$ для некоторого $\mu, \operatorname{cf} \mu \leqslant \omega$.

Единственное применение АС - доказательство 4) леммы 9 (и, как следствие, 3) теоремы 5); мне неизвестно, можно ли его избежать. Результаты о непротиворечивости остаются верными при предположениях, не добавляющих новых выигрышных множеств, таких, как "все множества сильно экстенсиональны" (см. [3], [6]), "не существует $\in$-циклов", "существуют наследственно невыигрышные множества любой структуры", "любое множество без $\in$-минимальных элементов имеет мощность больше любой данной”, и т.п. В целом, мы наблюдаем сильное различие нечетно- и четно-выигрышных множеств; последние более редки и причудливы.

\section{СПИСОК ЦИТИРОВАННОЙ ЛИТЕРАТУРЫ}

[1] T. J. Jech, Set Theory, The third millennium edition, revised and expanded, Springer Monographs in Mathematics, Springer-Verlag, Berlin, 2003. [2] T. E. Forster, Set Theory with a Universal Set, Exploring an Untyped Universe, Oxford Logic Guides, 31, The Clarendon Press, New York, 1995. [3] J. Barwise, L. Moss, Vicious Circles, On the Mathematics of Non-Wellfounded Phenomena, CSLI Lecture Notes, 60, CSLI Publ., Stanford, CA, 1996. [4] D. I. Saveliev (to appear). [5] M. Forti, F. Honsell, Ann. Scuola Norm. Sup. Pisa Cl. Sci. (4), 10:3 (1983), 493-522. [6] P. Aczel, Non-Well-Founded Sets, CSLI Lecture Notes, 14, CSLI Publ., Stanford, CA, 1988. [7] G. D'Agostino, Modal Logic and Non-Well-Founded Set Theory: Translation, Bisimulation, Interpolation, Ph.D. Thesis, ILLC Diss. Ser., 4, Institute for Logic, Language and Computation, Amsterdam, 1998. [8] L. Rieger, Czechoslovak Math. J., 7 (82) (1957), 323-357.

\section{Д. И. Савельев}

Поступило

Независимый московский университет, ВИНИТИ 12.05 .2004

E-mail: saveliev@mccme.ru, denissaveliev@mail.ru

Исправленный вариант

21.12.2006 